\title{
Pratique des ménages en matière d'hygiène et assainissement dans 3 villages du cercle de Kénieba dans la région de Kayes au Mali
}

\section{Practical of the households as regards hygiene and cleansing in 3 villages of the district of Kénieba in the area of Kayes in Mali}

\author{
AG IKNANE A ${ }^{1}$, SAKO Y², DJANGO DM ${ }^{3}$, DOUMBIA A ${ }^{2}$, AZIZ A $^{3}$. \\ ${ }^{1}$ Maître assistant en santé, Chef du service nutrition à l'Institut National de Recherche en Santé Publique - Bamako \\ 2 Maître assistant en anesthésie réanimation \\ 3 Médecin généraliste
}

\section{Résumé}

Introduction : Un accès insuffisant à une eau salubre, à des conditions d'hygiène et d'assainissement constituent par ordre d'importance, le troisième facteur de risque des problèmes de santé des populations dans les pays en développement avec comme conséquence des taux de mortalité élevé [2]. Ils causent d'énormes répercussions sur l'environnement, l'éducation et les activités économiques. La présente étude a été initiée dans le but d'étudier le niveau de connaissances et les pratiques des ménages en vue d'induire des un changement de comportement favorable à la santé.

Matériel et méthodes. Une étude transversale descriptive par sondage systématique dans le village de Kassama et exhaustive dans les villages de Nétékoto et Diantissa dans le cercle de Kéniéba a été réalisée du 3 au 7 novembre 2010. Près de 147 mères ou tutrices d'enfant de moins de 5 ans, des groupements masculins, féminins, des chefs de ménages, les enseignants avaient fait l'objet d'entretiens individuels et de focus groupe afin de déterminer le niveau de connaissances et de pratique en matière d'eau d'assainissement et d'hygiène.

Résultats: L'étude a montré que le paludisme a été observé dans $42,2 \%$ suivi de la diarrhée simple ou dysentériforme dans $23,1 \%$ et des infections respiratoires aigues avec toux et rhume dans 7,5\%. En cas de maladies, le premier recours aux soins a été le centre de santé communautaire dans $96 \%$ des cas.

Les principales sources d'approvisionnement en eau des ménages ont été les puits $(44,2 \%)$, les pompes $(24,5 \%)$ et le marigot $(8,8 \%)$ en saison pluvieuse. En saison sèche, les pompes $(35,4 \%)$ et les puits $(36,1 \%)$ ont constitué les principales sources d'approvisionnement.

Conclusion: Un faible niveau de connaissance et de pratique des ménages en matière d'hygiène et d'assainissement a été observé avec une faible scolarisation des mères. La faible disponibilité des puisards $(13,6 \%)$, la méconnaissance des méthodes de traitement de l'eau $(29 \%)$ et de transformation des ordures ménagères pourraient être corrigées à travers la formation des mères qui pourraient s'investir dans l'assainissement du milieu au moyen d'activités d'intérêts publics génératrices de revenus.

Mots clés : hygiène, assainissement, eau, enfants, femmes, santé.

\section{Summary}

Introduction: An insufficient access to a salubrious water, in conditions of hygiene and cleansing constitutes by order of importance, the third factor of risk of the problems of pubic health in the developing countries with like consequence of the high death rate [2 ]. They cause enormous effects on the environment, education and the economic activities. The present study was initiated with an aim of studying the level of knowledge and the practices of the households in order to induce change of behavior favorable to health.

Material and methods. A descriptive cross-sectional study by systematic survey in the village of Kassama and exhaustive in the villages of Nétékoto and Diantissa in the district of Kéniéba was carried out from the 3 to 7 November 2010. Nearly 147 mothers or tutors of child of less than 5 years, groupings male, female, heads of households, the teachers had been the object of individual talks and fosud group in order to determine the level of knowledge and practice as regards water of cleansing and hygiene.

Results: The study showed that paludism was observed in $42,2 \%$ follow-up of the simple diarrhoea or dysentéria in $23,1 \%$ and of the acute respiratory infections with cough and cold in $7,5 \%$. In the event of diseases, the first recourse to the care was the Community center of health in $96 \%$ of the cases.

The principal sources of supply water of the households were the wells $(44,2 \%)$, the pumps $(24,5 \%)$ and the backwater $(8,8 \%)$ in rainy season. In dry season, the pumps $(35,4 \%)$ and the wells $(36,1 \%)$ constituted the principal sources of provisioning.

Conclusion: A low level of knowledge and practice of the households hygiene cleansing was observed with a weak schooling of the mothers. The low availability of the sumps $(13,6 \%)$, the ignorance of the methods of water treatment $(29 \%)$ and of transformation of the household refuse could be corrected through the training of the mothers who could invest themselves in the cleansing of the medium by means of activities of public interests generating of incomes.

Key words: hygiene, cleansing, water, children, women, health.

\section{INTRODUCTION :}

L'assainissement et l'hygiène, joints à la consommation d'une eau salubre, sont essentiels pour maintenir un bon niveau de santé. Cependant, 2,6 milliards de personnes, 
soit $40 \%$ de la population mondiale, n'ont actuellement pas accès à un assainissement de base [1]. L'hygiène et l'assainissement insuffisants ont de graves conséquences sur la santé et d'énormes répercussions sur l'environnement, l'éducation et les activités économiques. L'accès insuffisant à une eau salubre, à l'assainissement et à l'hygiène est, par ordre d'importance, le troisième facteur de risque pour les problèmes de santé dans les pays en développement qui ont un taux de mortalité élevé $[2,3]$. A elles seules, les diarrhées sont à l'origine de 1,8 million de décès par an dans le monde et $90 \%$ de ces derniers sont ceux d'enfants de moins de 5 ans [2]. Cependant, il est admis que l'amélioration de la qualité de l'eau fait baisser de 15 à $20 \%$ la fréquence des diarrhées chez l'enfant [2,3] ; l'amélioration de l'hygiène, par le lavage des mains et la manipulation adéquate des denrées alimentaires, entraîne une baisse de $35 \%$ [2]; l'élimination sans danger des fèces des enfants s'accompagne d'une baisse de près de $40 \%$ [2,4] et enfin l'amélioration de l'hygiène et de l'assainissement peut potentiellement sauver énormément de vies. L'OMS déclare que dans les pays en développement $4 / 5$ de toutes les maladies sont causés par l'eau, et que dans le monde 1,1 milliards de personnes n'ont pas accès à une source d'eau potable [1]. Ceci est la cause de 3,4 millions de décès chaque année notamment des enfants [5].

La présente étude vise à évaluer le niveau de pratique des ménages en matière d'hygiène et assainissement au niveau des village de Kassama, Nétékoto et Diantissa dans le cercle de Kéniéba en vue de mettre en place un programme d'intervention dans ces localités.

\section{MATERIELS ET METHODES.}

Une étude transversale descriptive par sondage systématique au niveau du village de Kassama, et exhaustive dans les villages de Neketoko et Diantissa dans le cercle de Keniéba a été réalisée du 3 au 7 novembre 2009. Des entretiens individuels avec les mères d'enfants de moins de 5 ans ont été réalisés sur le niveau d'hygiène et assainissement au sein de leur ménage. Des discussions sous forme de focus groupe ont été réalisé auprès des conseillers de villages, des groupements de femmes, des élèves et des enseignants. L'observation directe sur le niveau d'hygiène et assainissement intra domiciliaire et dans au niveau des espaces fonctionnels des villages s'est effectuée pour mieux étayer les informations collectées au niveau individuel ou en groupe.

La taille de l'échantillon avait été calculée à l'aide de la formule de Daniel Schwarz [6]. La saisie des données a été réalisée à l'aide du logiciel EPI-DATA 3.0, et l'analyse au moyen du logiciel SPSS 17.0. Le test de chi deux de Person a été utilisé pour la comparaison des moyennes observées. Le consentement éclairé des autorités locales a été au obtenu au moment de la réalisation de l'enquête de terrain.

\section{RESULTATS}

Près de 96 de mères interrogées étaient mariées à Diantissa. A Kassama et Nétékoto respectivement $9 \%$ et
2,3\% de femmes étaient célibataires. Le niveau de scolarisation des mères était faible. A Kassama, seules $23,1 \%$ avaient atteint le niveau primaire contre $11,5 \%$ à Diantissa et Netekoto. Les principales maladies les plus fréquemment rencontrées au niveau des villages d'étude étaient dominées par le paludisme présumé ou Sumaya $(42,2 \%)$, la diarrhée simple ou dysentériforme $(23,1 \%)$ et les infections respiratoires aigues avec la toux et le rhume $(7,5 \%)$. Au niveau de l'ensemble de l'échantillon, $84,4 \%$ des enfants dormaient sous moustiquaire dont $73,5 \%$ avaient été imprégnées d'insecticide. Le taux de moustiquaires imprégné était de $81 \%$ à Diantissa, $74,4 \%$ à Netekoto et $70,5 \%$ à Kassama. Dans l'ensemble de l'échantillon, la principale source d'approvisionnement des ménages en eau potable pendant la saison sèche a été dominée par l'eau des puits traditionnels dans $36,1 \%$ et des forages dans $35,4 \%$. Seul $29,3 \%$ de ménages de l'échantillon traitaient leur eau de consommation avec $39,7 \%$ à Kassama et $30,8 \%$ à Diantissa et $9,3 \%$ à Neteketo. Sur les $29,3 \%$ de ménages qui traitaient l'eau de boisson, $63 \%$ d'entre eux utilisaient la technique de filtration et seulement $30 \%$ la javellisation. La technique de filtration a été utilisée par $61 \%$ de ménages de Kassama et $50 \%$ de ceux de Diantissa. Les latrines ont été utilisées comme lieu de défécation par 75,6\% de ménages à Kassama pendant que la nature a constituée le principal lieu de prédilection de la défécation pour $61,5 \%$ de ménages à Diantissa et $58,1 \%$ à Netekoto. Pour les ménages disposant de latrines, c'était les latrines traditionnelles avec une dalle en bois qui ont été utilisées. Les latrines traditionnelles avec dalles en ciment quant à elles n'ont été utilisées qu'au niveau de 6 ménages $(9,4 \%)$ à Kassama et un seul ménage $(7,1 \%)$ à Netekoto. A Diantissa par contre aucun ménage enquêté ne disposait de latrine avec dalle en ciment. Les fèces des enfants étaient dans la grande majorité des cas jeter dans les latrines à l'exception de Neteketo ou c'est plutôt dans la cour que les selles ont été versées $(90,7 \%)$. La devanture de la cour a constituée le lieu de prédilection pour jeter les ordures ménagères.

Dans l'ensemble de l'échantillon, la principale source d'approvisionnement des ménages en eau potable pendant la saison hivernale a été dominée par les puits traditionnels dans $44,2 \%$ suivi des forages avec $24,5 \%$. L'eau de mare ou de marigot a été utilisée par $8,8 \%$ de ménages en cette saison pluvieuse. Selon les localités, les puits traditionnels sont la principale source d'approvisionnement $(80,8 \%)$ pour la localité de Kassama, alors qu'à Diantissa et Neteketo les forages et les bornes fontaines sont les principales sources d'approvisionnement en eau.

\section{DISCUSSION}

Un échantillon de 147 mères d'enfants tutrices d'enfants de moins de cinq ans a été interrogé. La plus part des mères interrogées ne sont pas alphabétisées. Pour celles qui le sont, le niveau primaire prédomine avec $23 \%$ à Kassama et $11 \%$ dans les autres villages. Par rapport à l'état de santé, le paludisme a été observé dans $42,2 \%$ cas, précédé par la diarrhée, simple ou dysentériforme, dans $23,1 \%$ et les infections respiratoires aigu avec la 
toux et le rhume avec 7,5\%. Le taux d'utilisation de moustiquaire était de $84,4 \%$. Nos résultats sont supérieurs à ceux observés au niveau national par l'EDSM IV de 2006 [7] avec 41,4\% d'enfant de moins de 5 ans dormant sous moustiquaire. II reste également supérieur au niveau observé par la même étude au niveau rural avec 40,5\% [7]. La prévalence du paludisme chez les enfants qui était de $42,2 \%$ dans notre étude est nettement plus élevé que celle retrouvée par DOUMBIA A en 2009 dans la commune de Bemba qui était de $32,5 \%$ [8] et celle observée en 2006 par l'EDSM IV [7] avec un niveau de prévalence de la fièvre présumée palustre de $14,8 \%$ à Bamako et $24,2 \%$ à Kayes. Par contre, la prévalence était moins élevée que celle observée en 1996 par l'INRSP [9] à l'office du Niger avec $50 \%$ chez les enfants de 2 à 5 ans. L'enquête EDSM IV de 2006 [7] indique que $40,7 \%$ des enfants dorment sous moustiquaire au niveau national contre $26,9 \%$ à Kayes [7]. Nos résultats par rapport à la prévalence de la diarrhée chez les enfants sont supérieurs à ceux retrouvés pour la région de Kayes par les différentes enquêtes EDSM avec $20,8 \%$ en 2006 [7] et $22,8 \%$ en 2001 [10] et ceux de INRSP en 2001 qui trouvait un taux de $13,3 \%$ des enfants de moins 15 ans souffrant de diarrhée épisodique [11]. L'annuaire SLIS de la direction nationale de la santé en 2007 trouvait 10.088 cas de diarrhée chez les enfants de1-4ans dans la région de Kayes [12]. Ceci pourrait s'expliquer par la mauvaise pratique en matière d'hygiène et d'assainissement des ménages.

Notre étude a montré que les puits traditionnels constituaient la première source d'approvisionnement en eau potable avec $44,2 \%$ en saison pluvieuse et $36,1 \%$ en saison sèche. Ce taux est plus élevé que celui observé en 2009 dans cinq districts de santé de la région de Sikasso [13] avec $21 \%$ en saison sèche et $24,9 \%$ en saison pluvieuse. L'eau a été très souvent consommée directement sans traitement préalable par les ménages. L'eau de consommation n'a été traitée que par $29 \%$ des ménages, la javellisation de l'eau n'est utilisée que par $30 \%$ de ménages, elle n'est pas du tout connue à Nétékoto. De même, on note une forte possession de latrine dans la région de Kassama $75,6 \%$ par contre à Diantissa on a $34,6 \%$ et Nétékoto $34,9 \%$. Pour l'ensemble des ménages étudiés $46,9 \%$ ne se lavent les mains au sortir des toilettes qu'avec de l'eau simple, $29,7 \%$ se lavent toujours les mains au savon et $23,4 \%$ avec de l'eau et du savon. Par contre peu des ménages utilisent régulièrement le savon pour se laver les mains avant de manger avec seulement 31\% à Kassama, 21\% en Nétékoto, $0 \%$ à Diantissa. Cette situation mérite une large campagne d'information de sensibilisation en vu de mettre en relation le niveau d'hygiène des mains en particulier avec l'apparition de diarrhée chez les enfants.

\section{CONCLUSION}

L'étude met en évidence une bonne disponibilité des ménages en moustiquaire $(84,4 \%)$ et une bonne utilisation des moustiquaires imprégnés d'insecticides
$(73,5)$. Le faible niveau de scolarisation des mères pourrait avoir une répercussion sur le niveau de connaissance et de pratique en matière d'hygiène et assainissement des ménages. Des actions en faveur d'une plus grande disponibilité des ménages en puisards, de la formation aux méthodes de traitement de l'eau et de transformation des ordures ménagères pourraient réduire les maladies infantiles.

\section{REFERENCES BIBLIOGRAPHIQUES}

1. OMS. Eau, assainissement et santé, consulté 23/12/2009 à $19 \mathrm{~h} 30$ sur :

http://www.who.int/water sanitation health/publications/fact s 2004/fr/index.html

2. FONDS MONDIAL POUR L'ASSAINISSEMENT (GSF) : Approvisionnement en eau, hygiène et assainissement, consulté le 4/01/2010 à 9 h53 sur :

http://esa.un.org/iys/review09/global/pdfs/GSF_leaflet_Fren ch.pdf

3. APOU VP. Les maladies dues à l'eau dans les pays du tiers - monde, http://fig-stdie.education.fr/actes_2003/virapin/article.htm consulté le 3 mars consulté le 4/03/2010 à $17 \mathrm{~h} 30 \mathrm{mn}$

4. DIALLO C. Analyse des statistiques des maladies d'origine hydriques au Mali, Thèse de pharmacie 2006- 2007 FMPOS, $68 \mathrm{p}$.

5. AG IKNANE A. L'épidémiologie à la portée de tous Editions Jamana, 2006, 236p.

6. Croix Rouge Française. Eau, santé, éducation, consulté le 4/03/2010 à $17 \mathrm{~h}$ sur http://www.waternunc.com/fr/conf_eau_sante_edu_htm

7. Mali. Enquête Démographique et de santé du Mali, EDSM IV, CPS/Santé, DNSI, Macro International, Décembre 2007, $497 \mathrm{p}$.

8. DOUMBIA A. Evaluation du statut Nutritionnel des enfants de 6 à 59 mois dans la commune de Bamba (Cercle de Bourem, région de Gao), Thèse de Médecine 2008-2009, FMPOS, $89 \mathrm{p}$.

9. Diarra $M$, Ouattara $F$, Landouré $A$, Traoré $M$, Sangho $A$ et al. Etude environnementale de la zone de l'office de Niger, étude sur les aspects liés à la santé humaine, Novembre 1998, INRSP, $39 \mathrm{p}$.

10. UNICEF. Situation des enfants dans le monde, 1998. $79 p$.

11. INRSP. Consulté le 04/02/2010 sur http://www.ghmer.ch/activites_internationales_fr/INRS P.htm

12. MINISTERE DE LA SANTE. Annuaire SLIS 2007, DNS, $117 p$.

13. AG IKNANE, TRAORE Y, FOFANA A. Evaluation du statut nutritionnel des enfants de 0-59 mois et leurs mères dans la région de Sikasso, Novembre 2009, STC, 69 p.

14. MINISTERE DE LA SANTE. Profil national du Mali sur le statut environnement sain pour les enfants, Novembre 2004, DNS, 41p.

15. ABIDA M. Maladies liées à l'eau, MS, DNS/DHPS, Mars 2009, 26p.

16. UNICEF/OMS : Eau, assainissement, 17 juillet 2008 à Niamey consulté le 3/03/2010 18H10 sur : http://www.unicef.org/french/infobycountry/Niger_NewsLine .htm/ 